\title{
Detection and identification thresholds for consonant-vowel syllables
}

\author{
LOIS L. ELLIOTT, CHERYL LONGINOTTI, LUANN CLIFTON, and DIANNE MEYER \\ Northwestern University, Evanston, Illinois 60201
}

\begin{abstract}
Detection and identification thresholds were obtained for 6- and 10-year-old normal children and normal adults using five-formant synthesized consonant-vowel ([ba-[da\}-[ga]) stimuli. Sixyear-old children were found to have poorer detection than adults, just as they do for pure tones. For the identification task, the slopes of the performance-intensity functions were more shallow for 6-year-old children than for 10-year-olds and adults. Consequently, compared with 10-yearolds and adults, 6-year-old listeners require a greater increase in stimulus intensity above detection threshold to identify these stimuli at a high performance level. The influence of acoustic characteristics of the stimuli on all listeners is also discussed.
\end{abstract}

It has been reported that young children have poorer pure tone sensitivity than adults (e.g., Eagles, Wishik, Doerfler, Melnick, \& Levine, 1963; Elliott \& Katz, 1980a; Hartley \& Siegenthaler, 1964; Maxon, 1977; Roberts \& Huber, 1970; Roche, Siervogel, Himes, \& Johnson, 1978; Yoneshige \& Elliott, 1981). Higher intensities are also required for young children to identify monosyllabic words that are within their receptive vocabularies. For example, Elliott and Katz (1980b) tested normally hearing children on a fouralternative picture-pointing task. The test materials had been developed to be within the receptive vocabularies of inner city, 3-year-old children (Elliott, Conners, Kille, Levin, Ball, \& Katz, 1979). In order for the 3-year-olds to identify the auditory stimuli with near- $100 \%$ accuracy, the words had to be presented at a level approximately $20 \mathrm{~dB}$ higher than the level at which adults achieved this performance (Elliott \& Katz, 1980b). Five-year-old listeners required about $13 \mathrm{~dB}$ higher intensity than adults. In contrast, the performance of 10-year-olds did not differ significantly from adults'. This confirmed findings from previous studies (Elliott et al., 1979) in which the same words and pictures were used but the experimental procedure, subjects, and groupings of pictures all differed.

Other investigators, using a variety of stimuli and experimental procedures, have also reported agerelated differences in children's speech perception. However, relatively few other investigations have explicitly focused on age-related changes in children's word-identification performance when stimulus in-

This research was partially supported by an NSF grant. The authors thank Tim Smith for assistance in programming. Requests for reprints should be sent to Lois Elliott, Northwestern University, 2299 Sheridan Road, Evanston, Illinois 60201. tensity was the independent variable and stimulus item difficulty was carefully controlled. Jerger's (Jerger, Jerger, \& Lewis, 1981) work is an exception; she, too, found children's speech perception to be closely related to receptive vocabulary age. Greater attention has been given to developmental effects associated with voicing-onset-time (VOT) and temporal characteristics of the stimuli (Krause, 1978; Simon \& Fourcin, 1978; Tallal, 1981; Williams \& Bush, 1978; Zlatin \& Koenigsknecht, 1975). Developmental perceptual changes have also been demonstrated for synthetic voiced-stop consonants differing in place of articulation (Elliott, Longinotti, Meyer, Raz, \& Zucker, 1981). Many of these investigations have used computer-synthesized speech stimuli to permit precise control of the variable under investigation and to provide a continuum of small stimulus changes. We have been unable to identify any experiments that have both (1) focused closely on the stimulus intensity variable and (2) used very simple (e.g., consonant-vowel) speech stimuli. In view of the established intensity-related developmental effects for pure tone detection and identification of familiar words, there appeared to be a significant need for empirical data to address these issues.

The purpose of this experiment, then, was to compare the performance of children and adults on two speech perception tasks for which the speech stimuli had minimal meaningfulness or linguistic significance. Specifically, detection and identification of computer-synthesized consonant-vowel (CV) syllables that differed along the voiced-place dimension [ba, $\mathrm{d} a, \mathrm{~g} a$ ] were studied. It was expected, of course, that identification would occur at higher intensities than detection for all age groups. The question of major interest was whether smaller age-related performance differences would be obtained for the detection task than for the identification task. 


\section{METHOD}

\section{Stimuli}

The three CV syllables, [ba], [da], and [ga], were synthesized using an adaptation of Klatt's parallel-cascade synthesizer program (Klatt, 1980). Syllables 1 [ba], 7 [d a], and 13 [ga] from the set used by Elliott et al. (1981) were employed. Each had five formants with bursts and differed from the others in onset spectra, formant-transition directions, and durations. They were equalized in RMS energy over their full $300-\mathrm{msec}$ durations. Stimuli were presented monaurally through TDH-49 earphones with Grason-Stadler 001 cushions. Before the detection and identification tasks were introduced, stimuli were presented individually at a comfortable listening level and the subject was required to repeat orally what he or she had heard. This "open response set" procedure insured that the three CVs were perceived as the intended syllables.

\section{Detection Thresholds}

Detection data were obtained using a three-interval forced-choice task. A storage oscilloscope positioned in front of the subject marked intervals by displaying " 1 ," " 2 ," " 3 " in succession. The subject responded by pressing one of three buttons; correct responses were reinforced by displaying a smiling face on the oscilloscope under the number of the correct response. A simple, updown adaptive procedure (Levitt, 1971) was used with 2-dB step sizes. Each run began well above threshold and terminated after 20 reversals; the mean of the last 15 reversals was taken as the threshold. Detection thresholds were obtained for each syllable separately, and the order of syllables was counterbalanced across subjects in each group.

\section{Identification Thresholds}

Identification data were obtained by a method of constant stimuli. On each trial, one of the three CV stimuli was presented while a circle on the storage oscilloscope marked the observation interval. The subject pressed one of three response buttons to identify or label the stimulus; the circle merged into a smiling face to provide feedback for each correct response. Three stimulus presentations at each of several levels enabled the experimenter to determine the range of levels at which the complete run (10 trials/level) should be administered. The goal was to select a range of intensities such that each subject's identification performance ranged from below $50 \%$ to nearly $100 \%$ correct for each of the three intermixed CVs. Each of the CV syllables was presented at five intensities, in 5-dB steps, in a computer-controlled intermixed random order. Different ranges of intensities for each syllable were used when needed. The percentages of correct responses for each CV at each intensity level were automatically printed at the end of the run.

\section{Subjects}

Six- and 10-year-old normal-hearing children and young adults (nean age 26.6 years) participated, with 12 subjects per group. All subjects were required to meet several criteria to assure normal hearing function: (1) pure tone, air-conduction sensitivity better than or equal to $15 \mathrm{~dB} \mathrm{HL}$ (re ANSI, 1969) at the octave frequencies between $250 \mathrm{~Hz}$ and $4 \mathrm{kHz}$; (2) normal tympanograms and acoustic reflexes present (verified at the beginning of each test session to insure against temporary middle ear disorders); (3) performance of at least $90 \%$ correct on the NU-CHIPS Test (a clinical test of speech understanding that uses monosyllabic words as stimuli; Elliott \& Katz, 1980c) presented $40 \mathrm{~dB}$ above each subject's speech reception threshold (SRT); and, for children, (4) scores on the Peabody Picture Vocabulary Test (Dunn, 1965) that were no lower than 1 standard deviation (SD) below the age norm. Subjects participated in three experimental sessions, each lasting about $1 \mathrm{~h}$, and were reimbursed.

During the first session, the nonexperimental auditory tests were performed and subjects were familiarized with the identification task. During this familiarization procedure, the children were asked to name the orthographic symbols (letters) attached to the response box. All of the children correctly identified the symbols without assistance. When the detection procedure was introduced in the second session, a check was made to be certain that all children could read the numbers. During both the second and third session, subjects completed one detection run for each of the $\mathrm{CV}$ syllables and one identification run for the intermixed CVs. Order of these procedures was counterbalanced across days and across members of each age group.

\section{RESULTS}

\section{Detection}

Matched $t$ tests and correlations were run on the detection data to check for differences between performance in the second and the third sessions. The between-trials differences obtained for each syllable ranged from 4.5 to $5.5 \mathrm{~dB}$ for 6 -year-olds, 3.0 to $5.3 \mathrm{~dB}$ for 10 -year-olds, and 2.5 to $3.8 \mathrm{~dB}$ for adults. Since none of these differences was statistically significant, measures for each subject for each condition were averaged across sessions and entered into a split-plot factorial (SPF 3.3) analysis of variance (ANOVA; Kirk, 1968). No differences between syllables emerged (see Figure 1), but the ANOVA revealed a significant age effect for detection $[F(2,33)$ $=7.11, \mathrm{p}<.005$ ]. Multiple comparisons [honestly significant difference (HSD) test; Kirk, 1968] revealed that detection thresholds for 6-year-old children were significantly poorer $(p<.05)$ than those for both adults and 10-year-old children. The detection

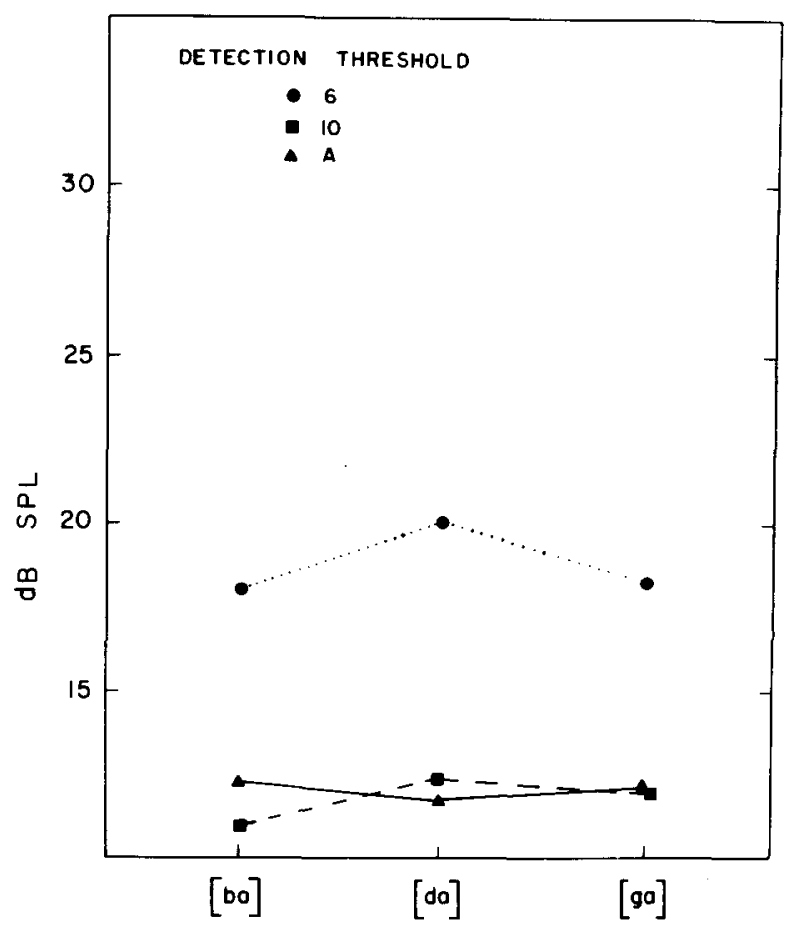

Figure 1. Levels required for $50 \%$ correct detection for each syllable and each age group. 
thresholds of 10-year-old children did not differ significantly from those of the adults. ${ }^{2}$

\section{Identification}

For each subject and for each of the three syllables separately, data were averaged over both sessions and a logistic function was fit by the method of maximum likelihood; the goodness of fit of the resulting function was tested by chi-square. Thus, for each subject, three separate functions (one for each syllable) were determined. From each of these functions, the point of $50 \%$ correct identification (here called identification threshold) and the slope of the function at the $50 \%$ point were determined and entered into separate SPF ANOVAs. In all except four cases (among 108 fitted functions), the logistic function was considered to be a very good fit $\left(\chi^{2}<3.84, p>.10\right)$. The four exceptions occurred for four different conditions (of age, syllable, threshold, and slope) and were handled by estimating a "missing data point."

Mean levels required for $50 \%$ correct identification are shown in Figure 2, which reveals significant effects for age $[F(2,33)=5.64, p<.01]$ and syllable $[F(2,66)=25.37, p<.001]$. Six-year-olds had identification thresholds that were significantly higher than those of both 10-year-olds and adults $(p<.05$, HSD test). There was no significant difference between identification thresholds of 10-year-olds and adults. Also, there was no significant interaction between age and syllable $[F(4,66)=.76]$.

Major differences in identification thresholds occurred among the three syllables. The intensity required for identification of syllables increased from

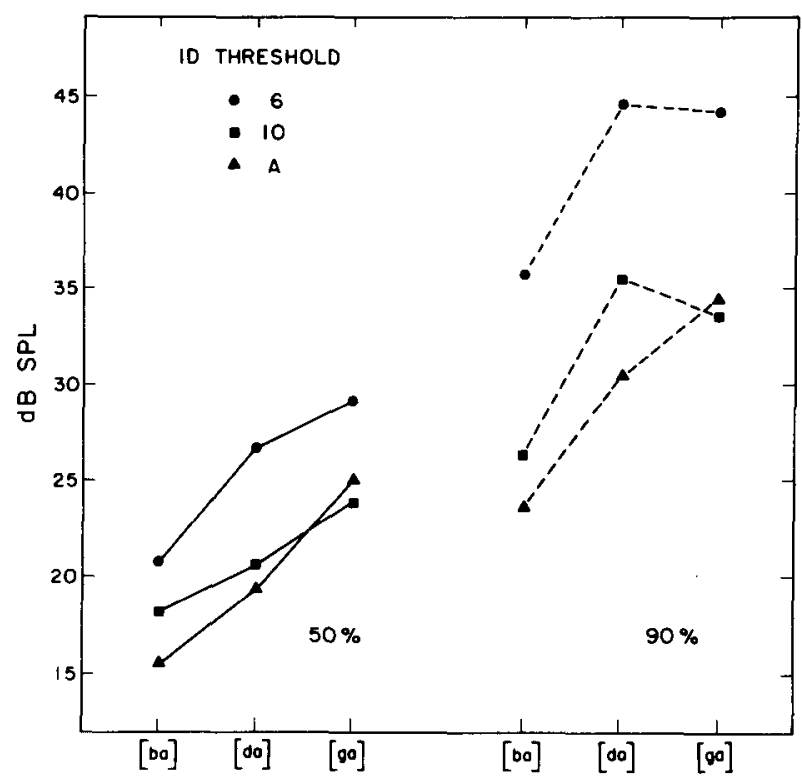

Figure 2. Levels required for $50 \%$ correct identification (left side of panel) and $90 \%$ correct identification (right side of panel) for each syllable and each age group.

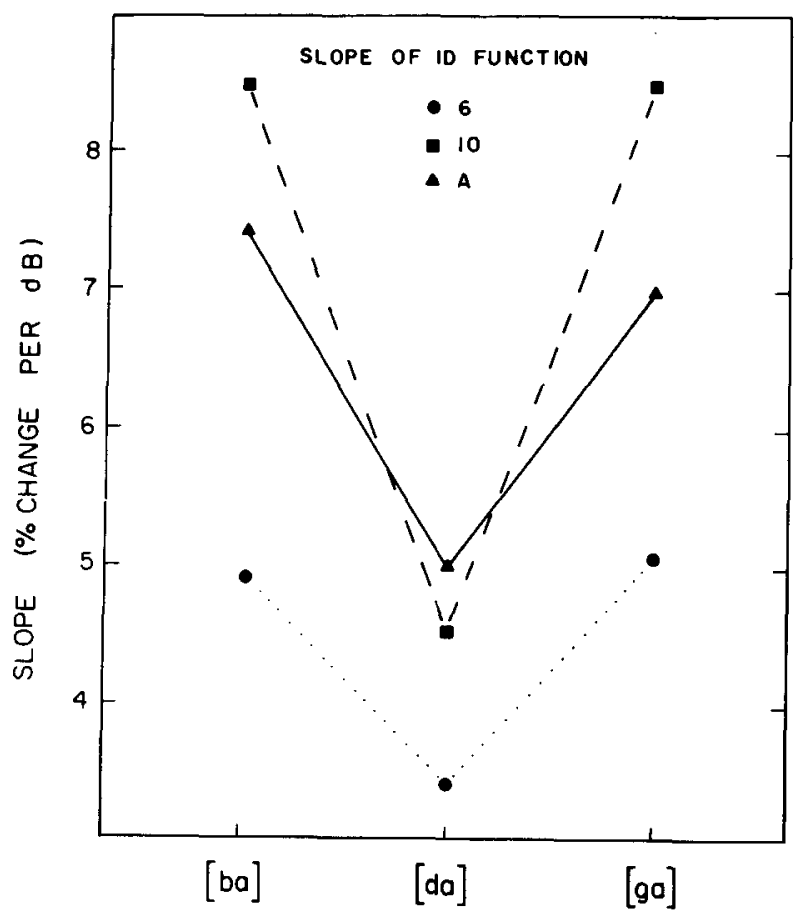

Figure 3. Average slopes of the identification functions at the $\mathbf{5 0 \%}$ correct point for each syllable and each age group.

$[\mathrm{b} a]$ to $[\mathrm{d} a]$ to $[\mathrm{g} a]-\mathrm{a}$ result that was consistent within each of the three subject age groups. Multiple comparisons (HSD test) revealed that the identification threshold for each syllable was significantly different $(p<.05)$ from the threshold for each of the other two syllables.

The slopes of the identification performanceintensity functions (Figure 3) differed significantly for both age $[F(2,33)=9.67, p<.001]$ and syllable $[F(2,66)=8.93, p<.001]$. The mean slope of the function for 6-year-old subjects was significantly more shallow than the slopes for both 10-year-olds and adults ( $p<.05$, HSD test). The mean slope for 10-year-olds was numerically greater than for adults, but the difference was not significant. The slope for [da] was significantly more shallow $(p<.05)$ than the slopes for both $[\mathrm{b} a]$ and $[\mathrm{g} a]$ for all ages. There was no significant age $X$ syllable interaction $[F(4,66)$ $=.68]$.

Detection and identification levels (both at $50 \%$ correct) were compared by matched $t$ tests for each syllable and each age group (Table 1). With one exception, identification threshold levels were significantly higher than detection thresholds, as would be expected. The matched $t$ test for 6-year-olds' [ba] failed to achieve statistical significance because of within-sample variability for both tasks. (Intersubject variability was approximately the same for both tasks, within each of the three age groups.) These 
significant differences confirm that subjects were responding differently to the two experimental tasks.

Because the slopes of the performance-intensity functions for the identification task differed across ages, the identification thresholds could not readily be compared with earlier data (Elliott \& Katz, 1980) representing asymptotic perception of monosyllabic words. Therefore, the intensities required for $90 \%$ correct identification of each syllable by each age group were estimated from the derived functions. These results, also plotted in Figure 2, show larger age differences than the $50 \%$ correct values, particularly between 6- and 10-year-olds' performances (as would be expected on the basis of more shallow identification slopes for the youngest subjects). Also, the points for 10-year-olds and adults show a larger decibel difference for [da]. These age differences in slopes lead to results that are of approximately the same magnitude as those Elliott and Katz (1980b) observed for identification of simple monosyllabic nouns.

A final analysis was completed in which, for each subject and each syllable, the difference was obtained between detection and identification thresholds (both $50 \%$ correct points). This value might be thought of as a type of sensation level (SL, the intensity increment above the detection threshold) that was needed to attain the identification threshold. Table 2 indicates essentially no age-related differences in these intensity increments for the [d $a$ ] and [ga] syllables; for $[\mathrm{b} a]$ there were no significant differences either, although 10-year-olds required a slightly larger increment than did either 6-year-olds or adults. However, there were consistent differences among syllables in the intensity increments required for $50 \%$ identification $[F(2,66)=28.11, p<.001)$. HSD tests showed

Table 1

Comparison of Levels Required for 50\% Correct Detection and Identification (Matched $t$ Tests)

\begin{tabular}{rllr}
\hline & {$[\mathrm{b} a]$} & {$[\mathrm{d} a]$} & \multicolumn{1}{c}{$[\mathrm{g} a]$} \\
\hline 6 & 1.15 & $2.23^{* *}$ & $4.46 \dagger$ \\
10 & $4.72 \dagger$ & $4.91 \dagger$ & $11.76 \dagger$ \\
$\mathrm{A}$ & $2.74^{*}$ & $5.24 \dagger$ & $5.03 \dagger$ \\
\hline
\end{tabular}

${ }^{*} p<.01 . \quad * *<<.025 . \quad t p<.001$.

Table 2

Mean Sensation Levels (Means of Individual Differences Between 50\% Detection Level and 50\% Correct Identification)

Syllable

\begin{tabular}{lcccc}
\cline { 2 - 4 } Age & {$[\mathrm{b} a]$} & {$[\mathrm{d} a]$} & {$[\mathrm{g} a]$} & Mean \\
\hline 6 Years & 4.2 & 7.8 & 11.6 & 7.9 \\
10 Years & 7.1 & 8.6 & 12.3 & 9.3 \\
Adults & 3.5 & 6.9 & 13.1 & 7.8 \\
Mean & 4.9 & 7.8 & 12.3 & \\
\hline
\end{tabular}

Table 3

Relative RMS Levels (in Decibels) Measured from Waveform Onset to Various Points Within the Stimulus

\begin{tabular}{rrrr}
\hline$\Delta \mathrm{t}$ & {$[\mathrm{b} a]$} & {$[\mathrm{d} a]$} & {$[\mathrm{g} a]$} \\
\hline 8 & 4.3 & .0 & 7.5 \\
12 & 20.0 & 1.1 & 9.0 \\
19 & 23.2 & 17.3 & 9.4 \\
52 & 29.4 & 26.4 & 23.3 \\
79 & 29.9 & 27.7 & 24.5 \\
300 & 28.9 & 28.9 & 29.0 \\
\hline
\end{tabular}

Note- $\Delta t=$ time (in milliseconds) over which RMS level is obtained $(300 \mathrm{msec}=$ full duration). All levels are reported relative to the first $8 \mathrm{msec}$ of $/ \mathrm{da} /$.

that the increment required for [da] was significantly higher than that needed for $[b a](p<.05)$, and the increment required for $[\mathrm{g} a]$ was greater than that for either of the other two stimuli $(p<.05)$. This probably relates to acoustic characteristics of the stimuli. Stimuli had identical total durations and were equalized in RMS energy. However, because the durations of the consonant formant transitions decreased from $[\mathrm{g} a]$ to $[\mathrm{d} a]$ to $[\mathrm{b} a]$, as occurs in natural speech, the relative intensities of the moving formant portions of the syllables were not equal. Measurements of the energy within the initial portions of these syllables revealed the differences shown in Table 3. These RMS voltage measurements of the electrical signals were all made from waveform onset to a point within the waveform, here noted as $\Delta t$. Thus, during the first $8 \mathrm{msec}$ of the signals, [ga] had the highest RMS-dB value and [d $a$ ] the lowest. During the first $52 \mathrm{msec}$, however, $[\mathrm{b} a]$ had greatest RMS-dB level and [ga] the least. This latter situation held throughout the remainder of the formant transition period. (The difference in RMS-dB levels for the full durations of the stimuli was less than $.2 \mathrm{~dB}$.) Thus, the higher intensity required for the identification threshold of [ga] as compared with [ba] may reflect RMS-dB differences for the initial portions of these syllables.

\section{DISCUSSION}

Age-related differences were obtained in this study for detection thresholds, for identification thresholds, for identification thresholds, and for slopes of the performance-intensity identification functions. The place dimension of the consonants did not lead to significant differences for detection but did for the identification task.

The age differences seen in the detection data (Figure 1) are consistent with those obtained in studies of pure tone detection in which auditory sensitivity of 6-year-old children has been found to be poorer than that of 10-year-olds and adults (e.g., Elliott \& Katz, 1980a; Yoneshige \& Elliott, 1981). It seems likely that the energy of the vowel $[a]$ dominated detection 
for the three CV syllables and accounted for the lack of detection differences across syllables. Exact reasons for age-related detection differences are not understood. Eagles and his co-workers (1963) attributed this type of result to greater prevalence of middle ear disorders in younger children, but that factor has been ruled out in this study by aural immittance testing. Yoneshige and Elliott (1981) demonstrated that neither poor earphone seal nor ear canal resonance was responsible for detection differences, and several studies (Elliott \& Katz, 1980a; Yoneshige \& Elliott, 1981) have shown that young subjects exhibit no greater response variability than do adults. One must conclude that age-related differences in detection are "true" differences and may relate to presently unspecified physiological or cognitive developmental changes.

The absence of age-related differences between detection and identification thresholds was unexpected, since previous work using a word-identification task had demonstrated large age-related performance differences when subjects were tested at equal sensation levels (Elliott \& Katz, 1980b). The difference between the two sets of results may reflect the linguistic complexity of the stimuli (i.e., meaningful words vs. nonsense syllables), the larger number of response alternatives, and age-related differences in integrating acoustic information with the visual information present in the picture-response alternatives of the word recognition task. In any case, the finding of approximately equal SLs required for $50 \%$ correct identification for the three age groups does not necessarily imply a single developmental factor underlying the age difference in both detection and identification. That is, the task demands for detection and identification were considerably different even though both involved a choice among three response alternatives.

The statistically significant differences for both age and syllable in the analysis of slopes of the performance-intensity functions for identification are of interest. (Since performance-intensity functions were not obtained for the detection task, it is not possible to determine whether slopes for that condition also varied across age groups.) The differences in slopes for syllables were consistent for each of the three age groups and are probably attributable to acoustic characteristics of the stimuli and the audibility of acoustic cues as intensity increases. As mentioned above, [da] had a lower RMS level at consonant onset than did the other two syllables. Also, Cullen and Collins (1978) have reported poor temporal integration for two-component falling tone glides when the signals are of short durations. This type of phenomenon may partially explain the very shallow slopes for $[\mathrm{d} a]$, compared with $[\mathrm{b} a]$ and [ga], since the major information-bearing formants at consonant onset of [da] both fall. The age effect for slopes of the identification functions means that 6-year-old listeners do not derive as much information as older subjects, as stimulus intensity increases. Nonmonotonic identification functions of the type recently described by Dorman and Dougherty (1981) for two-formant syllables were never seen in the present study; this difference may relate to the fiveformant structure of the CVs used here, as compared with the less-natural-sounding CVs of Dorman and Dougherty's work.

In summary, results reported in this paper indicate that 6-year-old children have poorer detection for voiced stop-consonant $\mathrm{CV}$ syllables, just as they do for pure tones. When identification thresholds of these CVs are measured at the $50 \%$ correct level, to correspond with the procedure most frequently used for assessing detection, subjects in the three age groups reach this performance point at essentially the same sensation levels. The significant differences in slopes of the identification functions clearly indicate, however, that identification performance of 6-year-old listeners is poorer than performance of 10-year-olds and adults-and that intensities required for asymptotic performance levels of the sort usually obtained in clinical testing would reveal larger developmental differences.

\section{REFERENCES}

Cullen, J. K., \& Collins, M. J. Temporal integration of twocomponent tone glides. Journal of the Acoustical Society of America, 1978, 64, 1526-1527.

Dorman, M. F., \& Dougherty, K. Shifts in phonetic identification with changes in signal presentation level. Journal of the Acoustical Society of America, 1981, 69, 1439-1445.

Dunn, L. M. Peabody Picture Vocabulary Test. Circle Pines, Minn: American Guidance Service, 1965.

Eagles, E. L., Wishik, S. M., Doerfler, L. G., Melnick, W., \& Levine, H. S. Hearing sensitivity and related factors in children. St. Louis: Laryngoscope Press, 1963.

Elliott, L. L., Connors, S., Kille, E., Levin, S., BAL, K., \& KATz, D. R. Children's understanding of monosyllabic nouns in quiet and in noise. Journal of the Acoustical Society of America, 1979, 66, 12-21.

Ezliotr, L. L., \& Katz, D. R. Children's pure-tone detection. Journal of the Acoustical Society of America, 1980, 67, 343344. (a)

ELLIOTT, L. L., \& KATZ, D. R. Development of a new children's test of speech discrimination. St. Louis: Auditec, 1980. (b)

Elliotr, L. L., \& KATz, D. R. Northwestern UniversityChildren's Perception of Speech Test. St. Louis: Auditec, 1980. (c)

EllotT, L. L., Longinotti, C., Meyer, D., RAz, I., \& Zucker, K. Developmental differences in identifying and discriminating CV syllables. Journal of the Acoustical Society of America, 1981, 70, 669-677.

Hartley, H. V., \& Siegenthalen, B. M. Relationships between Békésy fixed frequency and conventional audiometry with children. Journal of Auditory Research, 1964, 4, 15-22. 
Jerger, S., Jerger, J., \& Lewis, S. Pediatric speech intelligibility test. II. Effect of receptive language age and chronological ages. International Journal of Pediatric Otorhinolaryngology, 1981 , in press.

Johnson, D. M., Watson, C. S., \& Kelley, W. J. Interval biases in 3 AFC experiments. Journal of the Acoustical Society of America, 1980, 68, S60.

KIRK, R. E. Experimental design: Procedures for the behavioral sciences. Belmont, Calif: Brooks/Cole, 1968.

KIATT, D. H. Software for a cascade/parallel formant synthesizer. Journal of the Acoustical Society of America, 1980, 67, 971-995.

KRAU8E, S. E. Developmental use of vowel duration as a cue to postvocalic consonant voicing: $A$ perception and production study. Unpublished doctoral dissertation, Northwestern University, 1978.

LEviTT, H. Transformed up-down methods in psychoacoustics. Journal of the Acoustical Society of America, 1971, 40, 467-477.

Maxon, A. B. Psychoacoustic performance in children: $A$ developmental study. Unpublished doctoral dissertation, City University of New York, 1977.

Robert8, J., \& Huber, P. Hearing levels of children by age and sex. Washington, D.C: National Center for Health Statistics, 1970.

Roche, A. F., Siervoael, R. M., Himes, J. H., \& Johnson, D. L. Longitudinal study of hearing in children: Baseline data concerning auditory thresholds, noise exposure, and biological factors. Journal of the Acoustical Society of America, 1978, 64, 1593-1601.

Simon, C., \& Fourcin, A. J. Cross-language study of speechpattern learning. Journal of the Acoustical Society of America, $1978,63,925-935$.

Tallal, P., \& Stark, R. Speech acoustic-cue discrimination abilities of normally developing and language-impaired children. Journal of the Acoustical Society of America, 1981, 69, 568-574.

Williams, L., \& Bush, M. Discrimination by young infants of voiced stop consonants with and without release bursts. Journal of the Acoustical Society of America, 1978, 63, 1223-1226.

Yoneshige, Y., \& ElliotT, L. L. Pure tone sensitivity and ear canal pressure at threshold in children and adults. Journal of the Acoustical Society of America, 1981, 70, 1271-1276.

Yost, W. A. A forced-choice adaptive procedure for measuring auditory thresholds in children. Behavior Research Methods \& Instrumentation, 1978, 10, 671-677.

Zlatin, M. A., \& Koenigsknecht, R. A. Development of the voicing contrast: Perception of stop consonants. Journal of Speech and Hearing Research, 1975, 18, 541-553.

\section{NOTES}

1. Throughout this paper, "threshold" is used to denote the level of $50 \%$ correct performance.

2. Johnson, Watson, and Kelley (1980) have recently found that, in a three-interval forced-choice procedure, detection is poorest for stimuli presented in the first observation interval and best for stimuli presented in the third interval. An analysis of detection data of this study revealed poorer performance for stimuli presented in the first interval but essentially no difference between those presented in the second and third intervals. Most importantly for this study, the effect was identical in magnitude for the three age groups.

(Manuscript received July 1,1981 ;

revision accepted for publication August 17, 1981.) 